\title{
Errors in the administration and use of medications
}

\section{Summary}

Medical errors and adverse events are responsible for around 98,000 deaths annually in the USA, increasing problems and costs, decreasing confidence in Health Care. We have reviewed errors and adverse events in different disciplines. It is important to note that errors in the administration of medicines, errors in diagnosis, in the management of infections, and surgical procedures must be considered. The causes and effects resulting from errors should be reviewed and strategies developed to reduce these events. The use of the five "c" model is suggested to reduce the error in the administration of medications.

This is a valuable topic that has deserved extensive debate, in order to provide patient safety, a fundamental reason for medical work. ${ }^{1-4}$ Medical interventions that should benefit patients sometimes cause harm. Errors in medical care can occur in various settings: hospitals, medical offices, nursing homes, pharmacies and patients' homes or homes, at any point in the treatment process, call it wrong medication, inappropriate treatment, or delay in exams laboratory. ${ }^{5}$ The error in the administration of medicines is defined as any foreseeable event that can be caused by the inconvenient use or lack of medication that may cause harm to the patient, with the medication being under the control of health professionals, patients or consumers). We must consider errors in prescription, administration or lack of compliance. A recent classification of medication errors divides them into lapses, lapses, and mistakes or violations. ${ }^{6}$ In retrospect we become aware of errors, but when we make them we are not aware that we are producing them.

\section{How can the problem cause of error be known?}

The causal problem can occur in various ways: due to inadequate or written communication (prescription, documentation, transcription), supply and storage (pharmacy, distribution error, or warehouse management), appreciation of the work overload, access, availability, staff status health (fatigue, stress), equipment problems (access, functionality), factors related to the patient (availability), interruptions, distractions during drug administration. Most studies identify individual responsibility for not having respected the reference framework as the primary cause of error. ${ }^{7}$ Avoiding medication errors is important by balancing what is written, giving a suitable medication for the patient's condition, within the limits created by the uncertainty regarding therapeutic decisions, in doses that optimize the balance of benefit to harm. In the prescribed balance, the mechanism of action of the drug should be linked to the pathophysiology of the disease. ${ }^{8}$

Medical error is a wrong concept, false judgment, or wrong action. The medical error is distinguished from the adverse event, in that the injury produced by the medical error defines the adverse event. Errors are often classified as adverse events, which makes it difficult to measure the incidence of errors in diagnosis. ${ }^{9-12}$ Medical error involves health professionals who have had any failure during patient care, causing some type of damage. In this medical act there is no bad faith and there is not always recklessness, inexperience or negligence that imply moral or judicial responsibility. Medical errors are often considered as human errors in health care. ${ }^{11,14}$ This medical error or human error occurs when a healthcare provider makes decisions, chooses an incorrect method of care, incorrectly
Volume 8 Issue 5 - 2020

\section{Miguel Oliveros Donohue}

Department of Pediatrics, San Marcos University, Peru

Correspondence: Miguel Oliveros Donohue, Department of Pediatrics, San Marcos University, Lima, Peru, Tel 05I-I4- 460-I689, Email drmigueoliveros@hotmail.com

Received: July 17, 2020 | Published: September 04, 2020

executes an appropriate method of care, mismanages medications, or mistakenly reads radio diagnostic aid images. Medication error is the second most common cause and can cause severe harm or even death. ${ }^{6}$

\section{Can the error alter the medical act?}

The beneficence expressed by medical knowledge, experience, skill and effort are aimed at recovering the health of patients, however, we must accept the error as a possibility that occurs sporadically. The error must be a source of learning, be commented on, discussed, and avoid its repetition. ${ }^{15}$ For this, it is necessary to be a cultivator of the truth, show humility and recognize that "error is human", that we must accept it as an unwanted fact, but unavoidable in problematic cases.

The improvement of teaching in undergraduate and graduate basic applied sciences, remembering that the contribution of knowledge must be accompanied by moral training, human communication, knowing the prevailing legislation, and requesting the support of the Ethics Committee to guide and solve controversies, will minimize his presentation.

\section{What impact does error have on health care?}

Studies carried out in the US and the UK estimate that mortality from adverse events of medical treatment resulting from errors is high, that the error is not an isolated event, but rather reflects system problems, and the cost is high. ${ }^{14,16}$ The number of people who die from adverse events is high, but it is difficult to measure the incidence of diagnostic errors, because they are not classified as such. ${ }^{17}$ Errors cause between 44,000 and 98,000 deaths annually in the US. It is estimated that a quarter of them are preventable. ${ }^{10,13} \mathrm{~A}$ study published in 2019 shows an annual rate of death due to adverse events of medical treatment from 4180 in 1990, to 5180 in 2016 and in total during this period they were estimated 123,603 deaths for this cause. ${ }^{17}$

\section{Why is clinical safety necessary?}

Patient safety is a discipline of health care that emerged with the complexity of health care systems, and the increase in the number of harmful patients in health centers. ${ }^{19}$ Its objective is to prevent and reduce the risks, errors and damages suffered by patients during the provision of healthcare. This discipline tends to continuous improvement, based on learning from mistakes and adverse events. ${ }^{22,23}$

Adverse events are injuries related to health care, rather than complications of the patient's disease, prolong hospital stay, require additional diagnostic and therapeutic procedures, cause sequelae 
at discharge, and can cause death. It is a problem with a frequent tendency and It is preventable in a high percentage of cases. When an adverse event occurs, the important thing is not to find out who made a mistake, but how and why the systems failed. ${ }^{21}$

Adverse events due to unsafe care are probably one of the top 10 causes of death and disability in the world. $50 \%$ of the causes of damage are preventable. The most damaging errors are related to the diagnosis, prescription and use of medications..$^{18}$

\section{What do they tell us about error and adverse events in surgery?}

Surgery is a dynamic, high-risk specialty. The investigation of adverse events and errors has mainly focused on estimating technical skills, but it is important to take into account decisions and teamwork. Check-up systems have been developed to provide patient safety and prevent errors and adverse events. ${ }^{20,21}$ A project of a multimodal work strategy for the surveillance and control of the safety of the surgical patient, to promote the culture of safety in the surgical field through training, learning from mistakes, and teamwork, to propose a change in the Routine healthcare, based on the application of preventive measures solidly supported by scientific evidence, has been initiated in Spain. ${ }^{22}$

\section{What measures should be taken to reduce the error in the administration of medicines?}

The ideal is to administer the correct dose of the correct medication, to the correct patient, through the correct route at the correct time. This model is called the five "c's". Currently, it is considered that in order to improve therapeutic safety, safe drugs should be developed, failsafe systems must be created with security measures and devices to minimize errors, and if they do occur, they do not reach have negative consequences for patients. ${ }^{23}$

\section{Can ethics modify the handling of medical error?}

Ethics, professional policy, and the law, as well as empirical works in the literature, suggest that the reporting of errors and adverse events should be a practical standard. Naivete about error may lessen rather than increase medical-legal responsibility for healthcare, and help alleviate patient concern. ${ }^{24}$ Disclosing medical errors to patients and family members serves as a catalyst for litigation and sets appropriate moral and ethical standards, which cannot be ignored when solutions are added to remedy the problem. ${ }^{25}$

From the ethical point of view, the confrontation of errors is masked in the "principle of beneficence" that promotes the wellbeing of patients, avoiding any act that may cause harm "principle of non-maleficence". The truth is an ethical imperative and errors must be revealed, disclosed, made known, reported with transparency in safeguarding medical ethics.

\section{Conclusion}

Errors have been considered in: the administration of medications, in the diagnosis, in the management of infections and in surgical procedures. The most damaging errors are related to the diagnosis, prescription and use of drugs. Medication error is the second most common cause of harm. Creating a culture of safety that involves reporting errors in the administration of drugs, will allow to fully address the Medication Cycle. The use of the five "c" correct doses, correct medication, correct patient, correct route and correct time has been suggested to reduce the possibility of medication administration errors.

\section{Acknowledgments}

None.

\section{Conflicts of interest}

Author declares that there is no conflict of interest.

\section{References}

1. Ceriani Cernadas JM. El error en medicina: Reflexiones acerca de sus causas y de una actitud mas critica en nuestra profesión. Arch Argent Pediatr. 2001;99(6):522-529.

2. Abubakar AR, Chedi B, Simbak N, et al. Medication error: The role of health care professionals, sources of error and prevention strategies. Journal of Chemical and Pharmaceutical Research. 2014;6(10):646-651.

3. Wittich CHM, Burkle CHM, Lanier WL. Medication errors: An overview for clinicians. Mayo Clinic. 2014;89(8):1116-1125.

4. Aguilar Sierra LE. Una mala decision te lleva al error medico. Revista Mexicana Anestesioloigia. 2018;41(S1):87-88.

5. Special Eurobarometer 241. European Comission. Medical error; 2006. $62 \mathrm{p}$.

6. William Dip. Medication errors. J R Coll Physicians. 2007;37(4):343-346.

7. Keers R, Williams SD, Cooke J, et al. Causes of medication administration Errors in Hospitals. Systematic Review of Quantitative and Qualitative Evidence. Drug Saf. 2013;36(11):1045-1067.

8. Aronson JK. Medications errors: What they are, how they happen, and how to avoid them. QJM. 2009;102(8):513-521.

9. Special Eurobarometer 241. European Comission. Medical error; 2006. $62 \mathrm{p}$.

10. Graber ML. The incidence of diagnostic error in medicine. BMJ Qual Saf. 2013;22(Suppl 2):ii21-ii27.

11. Alvarado Guevara AT, Flores Sandi G. Errores medicos. Acta Medica costarricense. 2009:51(1):16-23.

12. Kohn LT, Corrigan J, Donaldson Molla S. eds. Institute of Medicine Committee on Quality of Health Care in America. To err in Human Building a Safer Health System. Washington DC: National Academy Press; 2000.

13. Lunevicius R, Haagsa J. Incidence and mortality from adverse effects of medical treatment in the UK 1990-2013: Level, trends, patterns and comparisons. International Journal for Quality in Health Care. 2018;30(7):558-564.

14. Hayward Rodney A, Hofer TP. Estimating hospital deaths due to medical errors: preventability is in the eye of the reviewer. JAMA. 2001;286(4):415-420.

15. Wusthoff CJ. Medical mistakes and disclosure: The role of the medical student. JAMA. 2001;286(9):1080-1081.

16. National Academy of Science. Medication errors injure 1.5 million people and cost billions of dollars Annualy; 2006.

17. Sunshine JE, Meo N, Kassebaum NJ, et al. Association of adverse effects of medical treatment with mortality in the United States. A secondary analysis of the Global burden of diseases, Injury and risk factors Study. JAMA Network Open. 2019;2(1):e187041.

18. OMS. Seguridad del paciente; 2019. 
19. Taller de Seguridad Ciudadana. Magnitud del problema y sus causas. II Congreso Ibérico de Medicina interna: Oviedo; 2010.

20. De Vries EN, Hellman MW, Smorenburg SM, et al. Development and Validation of the surgical patient Safety System (SURPASS) Check list. Qual Self Health Care. 2009;18(2):121-126.

21. Sarker SK, Vincent CH. Errors in Surgery. International Journal of Surgery. 2005;3(1):75-81.

22. Programa de Cirugia Segura del Ssistema Nacional de Salud. Protocolo. Ministerio de Sanidad y Servicios socials: España; 2018. 111 p.
23. Otero MJ, Martin R, Robles MD, et al. Errores de medicacion. Sefh; 1998 $35 \mathrm{p}$.

24. Hebert PC, Levin AV, Robertson G. Bioethics for clinicians: 23. Disclosure of medical error. Canadian Medical Association. 2001;164(4):509-513.

25. Booney W. Medical errors: Moral and ethical considerations. Journal of Hospital Administration. 2014;3(2):80-88. 